\title{
Metabolic diversity in sweet potato (Ipomoea batatas, Lam.) leaves and storage roots
}

\author{
Margit Drapal', Genoveva Rossel ${ }^{2}$, Bettina Heider ${ }^{2}$ and Paul D. Fraser ${ }^{1}$
}

\begin{abstract}
Sweet potato (Ipomoea batatas, Lam.) is an important root vegetable in developing countries. After its domestication in Neotropical America, human migration led to the distribution of the sweet potato plant throughout the world. Both leaf and storage root are high in compounds of nutritional value. Yet, the storage roots are of particular value due to their significant content of provitamin A ( $\beta$-carotene). The breeding effort for elite sweet potato lines led to the reduction of genetic diversity and the potential to improve other traits. The focus of the present study was to assess the metabolic diversity of 27 sweet potato cultivars including landraces and improved varieties. A metabolite profiling approach was optimised for sweet potato leaf and storage root tissue and 130 metabolites identified with three different analysis platforms. The data highlighted a lack of correlation between storage root phenotype and leaf metabolism. Furthermore, the metabolic diversity of storage roots was based on the secondary metabolism, including phenylpropanoids and carotenoids. Three cultivars of three different flesh colouration (yellow, orange and purple) showed a significant difference of the primary metabolism. This data demonstrates the value of metabolite profiling to breeding programs as a means of identifying differences in phenotypes/chemotypes and characterising parental material for future pre-breeding resources.
\end{abstract}

\section{Introduction}

Sweet potato (Ipomoea batatas) is a herbaceous perennial vine, which produces storage roots and edible leaves and can grow on marginal lands. The sweet potato plants have been widely dispersed by humans throughout the world since its domestication in the New World ${ }^{1,2}$. Currently, sweet potato is the sixth most important food crop after rice, wheat, potatoes, maize and cassava. In 2015, 105 million tonnes of sweet potatoes were produced worldwide and $95 \%$ thereof in developing countries with China as the lead producer ${ }^{3}$.

Both the leaves and storage roots of sweet potato have a high nutritional value for the human diet. Next to starch which comprises $60 \%$ of the dry matter (DM), leaves and

\footnotetext{
Correspondence: Paul D. Fraser (P.Fraser@rhul.ac.uk)

'Biological Sciences, Royal Holloway University of London, Egham TW20 OEX, UK

${ }^{2}$ International Potato Center (CIP), CGIAR Research Program on Roots, Tubers and Bananas, Lima 12, Peru
}

storage roots are high in protein, dietary fibre, micronutrients (e.g. iron), vitamins (e.g. vitamin C) as well as bioactive compounds such as carotenoids and phenylpropanoids ${ }^{4-7}$. The main use of sweet potato leaves is as animal feed, as it can be harvested several times throughout the year, whereas storage roots are mainly for human consumption ${ }^{8}$. The storage roots of sweet potato vary in flesh colour from white to orange and to purple, depending on the nature of the pigments produced. The two major pigment classes in sweet potato are carotenoids, cream to orange colouration, and anthocyanins, reddish to bluish purple, and both classes are known for their antioxidant properties. Previous studies highlighted the especially high levels of $\beta$-carotene in orange varieties and led to the incorporation of sweet potato into the program to prevent vitamin A deficiency in Africa ${ }^{9}$. Since then, one of the main focus of sweet potato breeding was an increase of starch and DM whilst maintaining high provitamin A levels. 
The breeding with recurrent elite lines led to a narrowed genetic pool in the secondary domestication centres, Oceania, Asia and Africa ${ }^{10}$. In addition to this, the main mode of sweet potato dispersal is asexual propagation which leads to a natural reduction of the genetic pool due to a lack of gene exchange ${ }^{11}$. Hence, modern breeding approaches are assessing the diversity of breeding lines and germplasm collection as well as trying to identify the genes involved in starch and carotenoid production through mapping populations ${ }^{11-14}$. Part of the elucidation process for starch and carotenoid biosynthesis is metabolomics, a wholesome approach to the metabolome of the improved tissue. Changes in one metabolite are in general the result of a modulation of many metabolic pathways and might lead to the depletion of other important metabolite $(s)^{15}$. Hence, a metabolite profiling approach has been adapted in the present study to assess the metabolic diversity of leaf and storage root tissue of a sweet potato diversity panel (Table 1). The methods applied include three different platforms to monitor a broad range of metabolites with different biochemical properties.

\section{Results}

\section{Metabolite composition of sweet potatoes}

The simple extraction method resulted in the detection of 130 identified metabolites in leaf and storage roots (Supplementary File S1). The identified metabolites

Table 1 List of cultivars included in the diversity panel

\begin{tabular}{|c|c|c|c|c|c|}
\hline Cultivar code & CIP no. & Cultivar name & Country of origin & Biol. status & Root flesh colour \\
\hline G-0372 & 401205 & Unknown & Mexico & LandRace & Yellow \\
\hline G-0528 & $441724^{a}$ & Cuitzeo & Mexico & LandRace & Intermediate orange \\
\hline G-0615 & 401527 & Unknown & Ecuador & LandRace & Dark purple \\
\hline G-0882 & 400473 & Unknown & Columbia & LandRace & White \\
\hline G-1000 & 401434 & Chaco Morado & Venezuela & LandRace & Pale yellow \\
\hline G-1196 & 400300 & Roxinha & Brasil & LandRace & Pale yellow \\
\hline G-1320 & 400177 & Aguela Manuchia & Bolivia & LandRace & Pale yellow with purple-red spots \\
\hline G-1656 & 400103 & Senorita & Argentine & LandRace & Pale yellow with orange spots \\
\hline$G-4615$ & 441141 & So 83 & Solomon Islands & LandRace & Dark orange \\
\hline G-5507 & 401225 & Camote & Nicaragua & LandRace & Cream \\
\hline PT-0005 & 440001 & Resisto & USA & ImpVariety & Intermediate orange \\
\hline PT-0023 & 440025 & Xushu 18 & China & ImpVariety & Cream \\
\hline PT-0026 & 440031 & Jewel & USA & ImpVariety & Intermediate orange \\
\hline PT-0045 & 420014 & Jonathan & Peru & ImpVariety & Intermediate orange \\
\hline PT-0094 & 420027 & Zapallo & Peru & LandRace & Dark yellow with orange ring and spots \\
\hline PT-0198 & $440291^{a}$ & Kinabakap & Philippines & LandRace & Cream \\
\hline PT-0204 & 440170 & Kemb-37 & Kenya & LandRace & Dark cream \\
\hline PT-0309 & 440175 & AVRDC-CN 1038-16 & Taiwan & BredLine & Pale yellow with purple spots \\
\hline PT-0408 & 440132 & Beauregard & USA & ImpVariety & Intermediate orange \\
\hline PT-0435 & 440280 & $85002-103$ & Tonga & LandRace & Cream \\
\hline PT-0450 & 440286 & VSP 1 & Philippines & ImpVariety & Intermediate orange with purple spots \\
\hline PT-0451 & 440287 & VSP 3 & Philippines & ImpVariety & Intermediate orange \\
\hline PT-0456 & 440290 & Waimanalao & Philippines & LandRace & Dark cream \\
\hline PT-0458 & 440252 & Beerwah Gold & Australia & ImpVariety & Intermediate orange \\
\hline PT-0465 & 440262 & Chin Mi (Eun Mi) & Korea & ImpVariety & Pale orange \\
\hline PT-0489 & 440166 & Tanzania & Uganda & LandRace & Pale yellow \\
\hline PT-0561 & 441755 & IB 90.10 .20 & India & BredLine & Cream \\
\hline
\end{tabular}

BredLine and ImpVariety represent breeding line and improved variety, the in-progress and final stage of a cultivar, respectively

${ }^{\text {a }}$ These cultivars were not included in the metabolite study due to the plants producing fibrous roots 
comprised primary and secondary metabolites of which 66 were detected in both tissue types (Fig. 1, Supplementary File S2). The remaining metabolites were unique to storage root and leaf tissue independently, 47 and 17 components, respectively. A third of all identified metabolites were classified as part of the phenylpropanoid metabolism and included several reoccurring features detected by LC-MS with UV/vis analysis. These features could only be putatively characterised as flavonoids by their spectral properties at $260 \mathrm{~nm}$ and were included in the metabolite analysis under the " $260 \mathrm{~nm}$ " identifier.

The other two-thirds of detected metabolites were classified into the following groups: amino acids, sugars, intermediates of the TCA cycle, sterols, membrane precursors and isoprenoids (IPP derived). The highest proportion of detected metabolite classes on a content basis were represented by sugar with $\sim 85 \%$, followed by phenylpropanoids, intermediates of the TCA cycle and amino acids with $2-5 \%{ }^{7}$. Isoprenoids represented $4 \%$ in leaf and less than $1 \%$ in storage roots and all other compound classes comprised less than $1 \%$ in both tissues.

The two cultivars, G-0528 and PT-0198, of the proposed diversity panel did produce fibrous roots and were suspected to carry a viral infection. The analysed material of both leaf and storage roots of these two cultivars were metabolically very different from the other samples, and hence were excluded from any further analysis/discussion of the diversity panel.

\section{Carotenoid and anthocyanin profiles in leaf and storage root}

The main pigments in the storage roots of sweet potato were identified as anthocyanins and carotenoids. The two dominant anthocyanins were identified with LC-MS/MS and comparison to literature as peonidin-3(6" -caffeoyl-6" p-hydroxybenzoyl-sophoroside)-5-glucoside and cyanidin-3(6"caffeoyl-6" -p-hydroxybenzoyl-sophoroside)-5-

glucoside $^{16}$. The cultivar G-0615 had the highest levels of both anthocyanins $\sim 3.3$ and $\sim 6 \mathrm{mg} / \mathrm{g}$ dry weight (DW), respectively. The cyanidin derivative was only detected in one other cultivar, G-1320 with $\sim 1 \mathrm{mg} / \mathrm{g}$ DW, whereas the peonidin derivative, known to be the most abundant anthocyanin in sweet potato, was detected in several cultivars, G-1320, G-1196, PT-0309, PT-0450 and PT0561; all containing $\sim 1 \mathrm{mg} / \mathrm{g} \mathrm{DW}^{17}$.

The carotenoids in storage roots were identified as phytoene, lutein, mutatochrome and $\beta$-carotene. Phytoene and lutein were only detected in cultivars with $\beta$ -

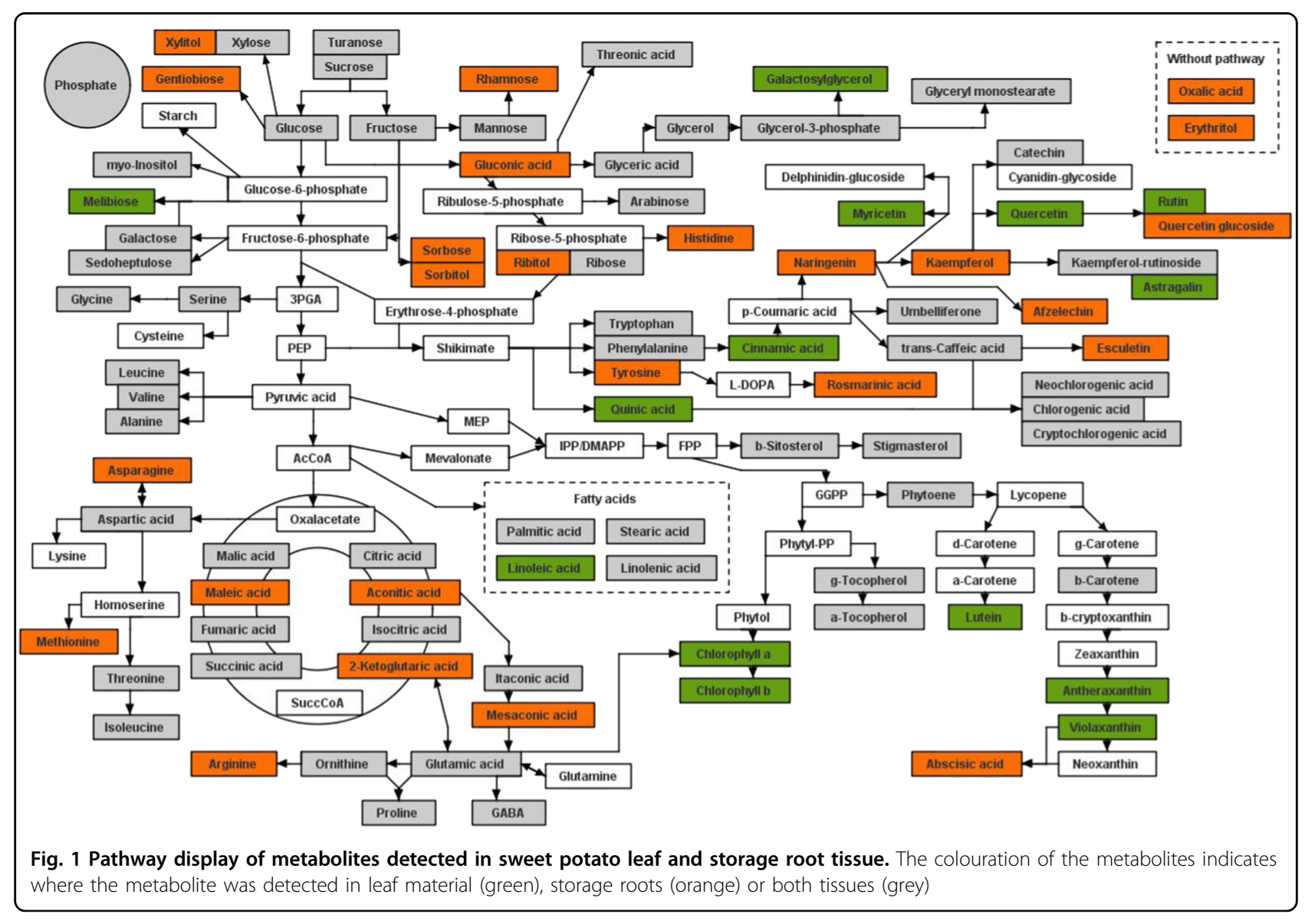




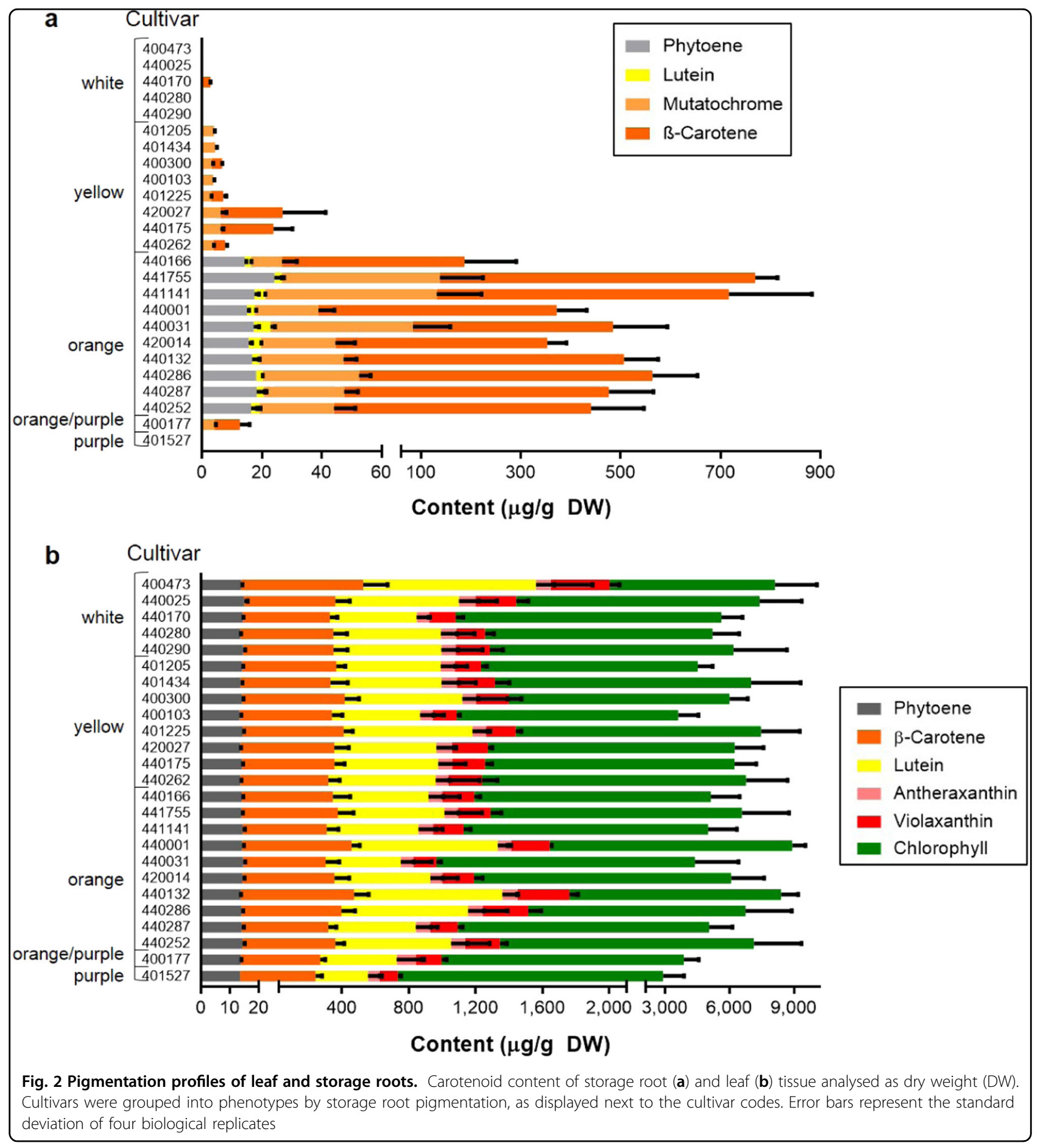

carotene levels $>160 \mu \mathrm{g} / \mathrm{g}$ DW (Fig. 2a). These ten cultivars also contained the highest levels of mutatochrome, a $\beta$-carotene 5,8 -epoxide ${ }^{18}$, ranging from 10 to $110 \mu \mathrm{g} / \mathrm{g}$ DW. The cultivar G-4615 contained the highest $\beta$ carotene and total carotenoid levels were $\sim 630$ and $\sim 800 \mu \mathrm{g} / \mathrm{g} \mathrm{DW}$, respectively. Five cultivars of the diversity panel contained no carotenoids and ten cultivars contained less than $30 \mu \mathrm{g} / \mathrm{g}$ DW of total carotenoids.
In the leaf material, the total content of carotenoids/ chlorophylls was 3-9 mg/g DW and chlorophyll constituted the main proportion (Fig. 2b). Neither the $\beta$ carotene nor the total carotenoid/chlorophyll content of the leaves could be correlated to the carotenoid content of the storage roots.

For the purpose of this study, the cultivars were grouped according to their carotenoid and specifically the $\beta$ - 


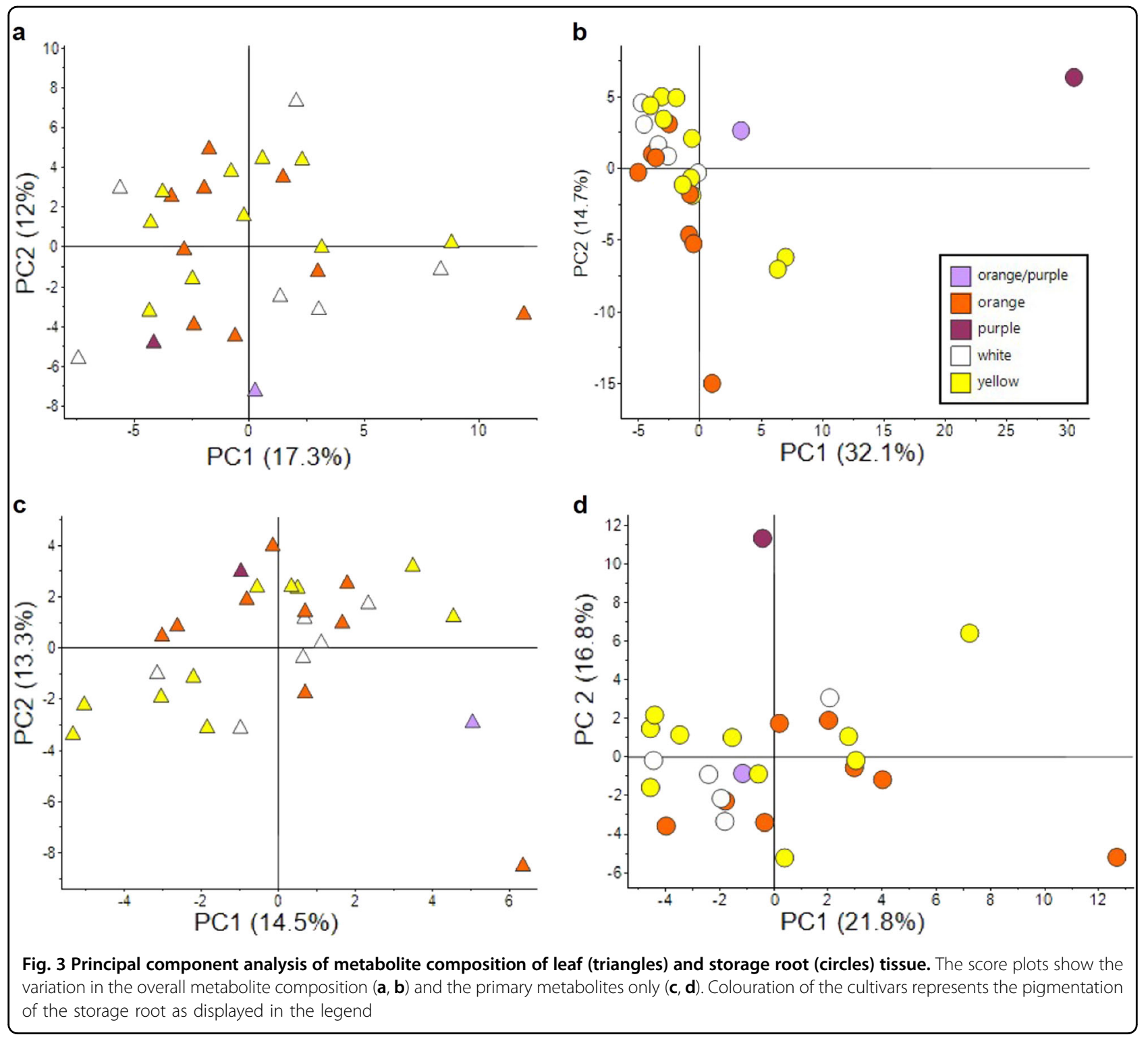

carotene content detected in the storage roots: white, yellow, orange, orange/purple and purple including five, nine, nine, one and one cultivars, respectively (Fig. 2a, b).

\section{Metabolic similarities of sweet potato leaves}

Principal component analysis (PCA) of the leaf material displayed no separation according to the phenotype of the storage roots. The score plots including all metabolites (Fig. 3a) or only primary metabolites (Fig. 3c) showed mixed clustering of the phenotypes. Analysis of variance (ANOVA) of leaf material detected six metabolites (5\% of total metabolite number) significant between phenotypes and about a third of metabolites significant between genotypes (Supplementary File S3). The significant metabolites between phenotypes included mainly phenylpropanoids and a fatty acid, whereas metabolites between genotypes comprised all compound classes including all isoprenoids except phytoene and $\beta$-carotene.

\section{Metabolic diversity of sweet potato storage roots}

The score plots of storage roots including all metabolites detected displayed clear cluster trends by phenotype (Fig. 3b), with white phenotypes located between the orange and yellow phenotypes. Contrary to this, the score plot based solely on primary metabolites showed a mixed cluster of all phenotypes (Fig. 3d). In both PCAs, the cultivars G-0615, G-4615 and PT-0309 were located away from the majority of cultivars. G-0615, the purple phenotype, had the highest levels of phenylpropanoids, several sugars and the amino acids aspartic acid, cysteine and GABA. The cultivar G-4615 had the highest carotenoid content and additionally the highest levels of half the 
amino acids detected. The yellow cultivar PT-0309 had the highest levels of cysteine, histidine and leucine as well as quinic acid and two sugars (Supplementary File S2).

A more detailed analysis of the storage root data by ANOVA indicated significant differences of isoprenoids, phenylpropanoids and sugars between phenotypes, of which isoprenoids were associated with orange cultivars and sugars with the purple phenotype. Half of the metabolites detected were significant between the phenotypes and over $80 \%$ of the total metabolites showed significance between the genotypes. The carotenoids had the highest significance values in both cases (Supplementary File S3).

Correlation analysis of the storage root metabolite data highlighted a positive correlation within compound classes, e.g. amino acids, phenylpropanoids and carotenoids (Supplementary Figure S1). Further positive correlations were detected between $\alpha$-tocopherol and phenylpropanoids; carotenoids and sedoheptulose, citric acid and malic acid; and catechin/epicatechin and anthocyanins. None of the negative correlations were significant.
The starch, sucrose and $\beta$-carotene content of the majority of varieties was evaluated with near-infrared reflectance spectroscopy (NIRS) in a previous crop and compared to the $\beta$-carotene measurement with ultraperformance liquid chromatography (UPLC) (Table 2). The $\beta$-carotene content established with NIRS showed similar results to the measurements with UPLC, except for some varieties with cream to light orange colour. This was to be expected as the colouration of the powder is comprised of carotenoids and phenolics, which can be clearly distinguished with the more accurate UPLC method. The linear regression between $\beta$-carotene and starch content in the storage roots indicated a significant negative correlation, whereas the correlation coefficient for sucrose and $\beta$-carotene indicated a low positive correlation (Fig. 4).

\section{Discussion}

The present trend in crop breeding is to improve nutritional quality and resilience whilst maintaining high yields ${ }^{19}$. To exploit the natural metabolic variation of

Table 2 Evaluation of starch, sucrose and carotenoid content in a selected number of cultivars

\begin{tabular}{|c|c|c|c|c|c|c|c|}
\hline Cultivar code & CIP no. & Root colour & Starch $^{\mathrm{a}}$ & Sucrose $e^{a}$ & $\beta$-Carotene ${ }^{a}$ & $\beta$-Carotene ${ }^{b}$ & Mutatochrome $^{\mathrm{b}}$ \\
\hline G-1656 & 400103 & Pale yellow with orange spots & 62.5 & 14.5 & 224.9 & 160.4 & 10.3 \\
\hline G-1320 & 400177 & Pale yellow with purple-red spots & 68.0 & 10.1 & 0.0 & 8.7 & 4.0 \\
\hline G-0882 & 400473 & Cream & 67.1 & 7.5 & 0.0 & 0.0 & 0.0 \\
\hline G-0372 & 401205 & Pale yellow & 70.4 & 6.3 & 88.7 & 0.0 & 4.1 \\
\hline G-0615 & 401527 & Dark purple & 66.0 & 8.9 & 0.0 & 0.0 & 0.0 \\
\hline PT-0045 & 420014 & Intermediate orange & 44.0 & 24.3 & 224.3 & 401.2 & 60.5 \\
\hline PT-0094 & 420027 & Dark yellow with orange ring and spots & 54.3 & 14.5 & 42.4 & 308.2 & 25.0 \\
\hline PT-0005 & 440001 & Dark orange & 53.2 & 17.9 & 576.7 & 585.2 & 110.4 \\
\hline PT-0023 & 440025 & Cream & 73.4 & 5.1 & 0.0 & 0.0 & 0.0 \\
\hline PT-0026 & 440031 & Intermediate orange & 59.5 & 11.4 & 270.5 & 332.8 & 21.2 \\
\hline PT-0408 & 440132 & Intermediate orange & 54.3 & 22.2 & 234.1 & 459.3 & 28.1 \\
\hline PT-0204 & 440170 & Cream & 69.6 & 5.6 & 0.0 & 2.8 & 0.0 \\
\hline PT-0309 & 440175 & Pale yellow with purple spots & 66.0 & 4.5 & 0.0 & 4.1 & 3.1 \\
\hline PT-0458 & 440252 & Intermediate orange & 63.1 & 10.3 & 392.6 & 396.3 & 24.8 \\
\hline PT-0465 & 440262 & Pale orange with yellow spots & 65.7 & N/A & 0.0 & 20.8 & 6.2 \\
\hline PT-0435 & 440280 & Cream & 60.7 & 17.1 & 295.2 & 0.0 & 0.0 \\
\hline PT-0450 & 440286 & Intermediate orange & 51.7 & 22.2 & 417.5 & 511.2 & 32.2 \\
\hline PT-0451 & 440287 & Intermediate orange & 56.1 & 14.1 & 324.1 & 428.8 & 26.9 \\
\hline PT-0456 & 440290 & Dark cream & 65.5 & 6.8 & 0.0 & 0.0 & 0.0 \\
\hline G-4615 & 441141 & Dark orange & 33.2 & 7.6 & 506.8 & 631.9 & 110.9 \\
\hline PT-0561 & 441755 & Dark yellow & 64.4 & 11.4 & 58.0 & 3.9 & 3.9 \\
\hline
\end{tabular}

Starch and sucrose are displayed as percentage of dry matter and carotenoid content was calculated as $\mu \mathrm{g} / \mathrm{g} \mathrm{DW}$

${ }^{a}$ Measurements were taken at CIP

${ }^{\mathrm{b}}$ Measurements were taken at RHUL 

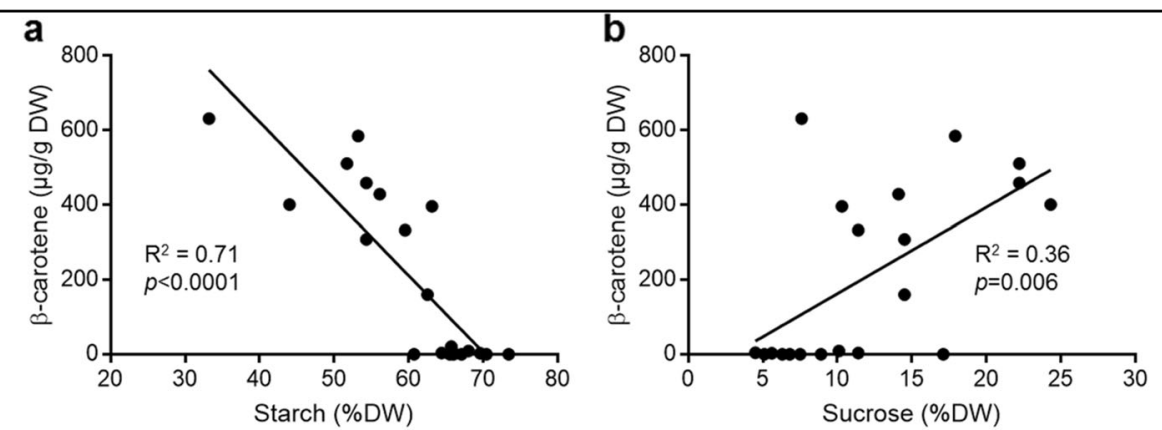

Fig. 4 Correaltion between B-carotene, starch and sucrose content in storage roots. Graph shows the linear regression between $B$-carotene measurements and starch (a) and sucrose (b) content. Linear regression coefficient $\left(R^{2}\right)$ and significance of the regression are displayed for each graph

sweet potato, the metabolite profiles of a diversity panel representing sweet potato phenotypes were elucidated.

One of the major results of the present study was the lack of correlation between leaf and storage root material, contrary to other crops, e.g. potato and yam ${ }^{20,21}$. This would suggest an independent genetic regulation between two tissue types of the same plant. However, the use of primary metabolites as a differentiator in either tissue displayed no separation between the storage root phenotype, country/region of origin or biological status as reported in genetic studies based on chloroplast and nuclear markers ${ }^{2,11,22,23}$. The metabolite data could reflect the reduced metabolic plasticity of sweet potato storage roots due to multiple recombination events not only in the region of domestication, Neotropical America, but also during the multiple distribution events of sweet potato into Oceania, Asia and Africa ${ }^{24}$. Additionally, the metabolite results support the hypothesis of incorporated transfer DNA from Agrobacterium spp. as part of the domestication process, as the landraces with known DNA fragments did not separate from the other cultivars ${ }^{25}$.

Another main discovery was the lack of correlation between carotenoid content and the majority of metabolites in storage roots. The presence of phytoene in orange phenotypes, solely indicates a higher phytoene synthase activity, whereas the correlation of carotenoids with sedoheptulose, citric and malic acid suggests only a minor increase of intermediates/precursors is necessary to support flux into the carotenoid biosynthesis. The metabolite data could therefore be the result of increased Orange gene $(\mathrm{Or})$ levels, providing more sink space/sites for stable carotenoid storage or the post-translational stabilisation of phytoene synthase. The latter effects could promote increased flux into the carotenoid pathway ${ }^{26,27}$.

Overall, the polyploidy of sweet potato and control of $\beta$ carotene levels via at least eight loci poses a challenge to determinate the exact processes involved in higher carotenoids content ${ }^{13}$. Yet, the inverse correlation trend between carotenoid and starch/sucrose content, ascertained on a small subset of the diversity panel (Table 2), has been published previously and supports the hypothesis that $\beta$-carotene and starch compete or infer with the formation of macromolecular structures in the same organelles ${ }^{13,28}$. The breeding of the desired starchy, dry and nutritious sweet potato highlighted the compromise between $\beta$-carotene and starch/DM content ${ }^{29,30}$. Surprisingly, the sucrose content had a low correlation with $\beta$-carotene which would indicate that the carotenoid content and sweetness of the root are unrelated. This is of great benefit for regions preferring sweet potato varieties with low sweetness consumer traits ${ }^{29}$. An example of a sweet potato combining the three traits, low sugar, high starch and high carotenoid levels, is the cultivar PT-0408, an improved variety originating from the USA. This cultivar has a $20 \%$ increase in starch content compared to a dark orange cultivar G-4615, and a similar sucrose content to a white cultivar G-0882, but still accounts for $~ 5 \%$ of the RDA for provitamin A per gram $\mathrm{DW}^{31}$.

The purple storage roots are in addition to the orange phenotype another source of antioxidants. Purple cultivars such as G-0615 have been reported with 13 times more of total phenolics compared to yellow or orange cultivars, which results in significantly higher radical scavenging activity ${ }^{6,32}$. Rodriguez-Bonilla et al. reported a difference between the leaf tissue of a purple cultivar from other phenotypes, which corresponded to the storage root but not the leaf material of the present study ${ }^{22}$. In both studies, only one purple cultivar was included and prompts a more detailed study of the whole metabolism of several purple phenotypes.

Summarising, the present study demonstrated the successful application of metabolite profiling to assess the metabolic diversity of sweet potato cultivars. The storage root data highlighted three cultivars, G-4615, PT-0309 and G-0615, which differed in their primary as well as secondary metabolite composition and represent suitable parental material for breeding efforts. The lack of metabolic diversity in all other cultivars analysed emphasise 
the importance of detailed genotyping, phenotyping and trait assessment to prevent further reduction of genetic diversity in elite breeding lines.

\section{Material and methods Plant material}

A total of 27 distinct clones were selected from the composite genotyping set to represent the range of diversity inherent in the sweet potato collection preserved in perpetuity at gene bank of the International Potato Center in Lima, Peru. Selection criteria were based on geographic origin, genetic distance and morphological traits such as flesh colour. In vitro plantlets were raised until sufficiently developed to take cuttings. Subsequently, cuttings were planted in $5 \mathrm{~L}$ pots in four repetitions and maintained under greenhouse conditions for 9 months in Lima, Peru.

\section{Measurements of starch, sucrose and $\beta$-carotene}

Data on starch, sucrose and $\beta$-carotene were derived from separate field experiments in 2013 and were provided by the Genoveva Rossel, sweet potato curator at the International Potato Center. NIRS was used to determine starch, sucrose and $\beta$-carotene in milled freeze-dried samples of sweet potato storage roots ${ }^{29}$. NIRS calibrations and validation were carried out ${ }^{33}$.

\section{Sample preparation and extraction}

Sweet potato leaf and storage root material was harvested, cleaned and immediately frozen in liquid nitrogen. Storage roots were rinsed in distilled water and cut into cubes before freezing. The frozen material was freezedried and ground to fine powder.

For metabolite analysis, a quality control, representing a pool of all samples, was created. All samples including the quality control were weighed $(10 \pm 0.5 \mathrm{mg})$ and extracted with a methanol-chloroform protocol ${ }^{34}$. Aliquots of the polar $(100$ and $650 \mu \mathrm{l})$ and the non-polar phase (650 and $700 \mu \mathrm{l})$ were dried down after extraction and stored at $-20{ }^{\circ} \mathrm{C}$ until analysis.

\section{Metabolite analysis and identification}

Aliquots of the non-polar phase were resuspended (leaf $100 \mu \mathrm{l}$; storage root $50 \mu \mathrm{l}$ ) in ethyl acetate and acetonitrile $(1: 9, \mathrm{v} / \mathrm{v})$ and analysed by UPLC. The diluted samples were analysed as previously described, identified by retention time and UV/vis light spectrum and quantified with dose-response curves for each isoprenoid ${ }^{34,35}$.

For gas chromatography-mass spectrometry analysis, the aliquots of the polar phase $(100 \mu \mathrm{l})$ were dried down with the internal standard $\mathrm{d}_{4}$-succinic acid $(5 \mu \mathrm{g})$ and the non-polar phase $(700 \mu \mathrm{l})$ with $\mathrm{d}_{27}$-myristic acid $(10 \mu \mathrm{g})$ and derivatised immediately before injection. The GC-MS analysis method included a 10:1 split mode. Identification of metabolites was based on retention time, retention indices and mass spectrum and the area quantified to the internal standard ${ }^{34,36}$

The polar extracts $(700 \mu \mathrm{l})$ for liquid chromatographymass spectrometry (LC-MS) were resuspended in methanol/water (100 $\mu \mathrm{l} ; 1: 1, \mathrm{v} / \mathrm{v})$ and an internal standard (genistein, $2.5 \mu \mathrm{g}$ ) added. After LC-MS analysis with a gradient of water/formic acid $(0.1 \%, \mathrm{v} / \mathrm{v})$ and methanol/ formic acid $(0.1 \%, \mathrm{v} / \mathrm{v})$, data analysis was performed using $\mathrm{R}$ package metaMS with a retention time window match set to $0.5 \mathrm{~min}^{20,36-38}$.

\section{Data analysis and statistical modelling}

Relative quantified data matrices were subjected to PCA using Simca P 13.0.3.0 (Umetrics, Sweden). Nonparametric one-way ANOVA after auto-scaling was performed using MetaboAnalyst $3.0^{39}$. UPLC results were displayed as bar charts using GraphPad Prism 7.02 (CA, USA). Metabolites were displayed as biochemical pathways constructed with BioSynLab software (www. biosynlab.com).

\section{Acknowledgements}

We would like to thank all donors who supported this research through their contributions to the CGIAR Fund: http://www.cgiar.org/about-us/ourfunders/. This research was undertaken as part of, and funded by, the CGIAR Research Program on Roots, Tubers and Bananas (RTB) and supported by CGIAR Fund Donors. The European Union Framework Program DISCO (from DISCOvery to products: a next-generation pipeline for the sustainable generation of high-value plant products; Project 613513) also contributed to this work.

\section{Conflict of interest}

The authors declare that they have no conflict of interest.

\section{Publisher's note}

Springer Nature remains neutral with regard to jurisdictional claims in published maps and institutional affiliations.

Supplementary Information accompanies this paper at (https://doi.org/ 10.1038/s41438-018-0075-5).

Received: 18 February 2018 Revised: 11 July 2018 Accepted: 1 August 2018 Published online: 01 January 2019

References

1. O'Brien, P. J. The sweet potato: its origin and dispersal. Am. Anthropol. 74, 342-365 (1972).

2. Roullier, C. et al. Disentangling the origins of cultivated sweet potato (Ipomoea batatas (L.) Lam). PLoS One 8, e62707 (2013).

3. CIP. Roots and tubers improving the lives of the poor. Annual Report (2015).

4. Mohanraj, R. \& Sivasankar, S. Sweet potato (Ipomoea batatas [L.] Lam)-a valuable medicinal food: a review. J. Med. Food 17, 733-741 (2014).

5. Shekhar, S., Mishra, D., Buragohain, A. K., Chakraborty, S. \& Chakraborty, N. Comparative analysis of phytochemicals and nutrient availability in two contrasting cultivars of sweet potato (Ipomoea batatas L.). Food Chem. 173, 957-965 (2015).

6. Teow, C. C. et al. Antioxidant activities, phenolic and $\beta$-carotene contents of sweet potato genotypes with varying flesh colours. Food Chem. 103, 829-838 (2007). 
7. Woolfe, J. A. Sweet Potato: An Untapped Food Resource (University Press, Cambridge, United Kingdom 1992).

8. Sun, H., Mu, T., Xi, L., Zhang, M. \& Chen, J. Sweet potato (Ipomoea batatas L.) leaves as nutritional and functional foods. Food Chem. 156, 380-389 (2014).

9. Burri, B. J. Evaluating sweet potato as an intervention food to prevent vitamin A deficiency. Compr. Rev. Food Sci. Food Saf. 10, 118-130 (2011).

10. Su, W. et al. Genome-wide assessment of population structure and genetic diversity and development of a core germplasm set for sweet potato based on specific length amplified fragment (SLAF) sequencing. PLoS One 12, e0172066 (2017).

11. Camargo, L. K. P., Mógor, A. F., Resende, J. T. V. \& Da-Silva, P. R. Establishment and molecular characterization of a sweet potato germplasm bank of the highlands of Paraná State, Brazil. Genet. Mol. Res. 12, 5574-5588 (2013).

12. Ahn, Y. O. et al. Exogenous sucrose utilization and starch biosynthesis among sweetpotato cultivars. Carbohydr. Res. 345, 55-60 (2010).

13. Cervantes-Flores, J. C. et al. Identification of quantitative trait loci for dry-matter, starch, and $\beta$-carotene content in sweetpotato. Mol. Breed. 28, 201-216 (2011).

14. Xie, F. et al. De novo sequencing and a comprehensive analysis of purple sweet potato (Ipomoea batatas L.) transcriptome. Planta 236, 101-113 (2012)

15. Ojong, P. B. et al. Variation of flavonoid content among sweetpotato accessions. J. Am. Soc. Hortic. Sci. 133, 819-824 (2008).

16. Cuevas Montilla, E., Hillebrand, S. \& Winterhalter, P. Anthocyanins in purple sweet potato (Ipomoea batatas L.) varieties. Fruit. Veg. Cereal Sci. Biotechnol. 5, 19-24 (2011)

17. $\mathrm{Xu}, \mathrm{J}$. et al. Characterisation and stability of anthocyanins in purple-fleshed sweet potato P40. Food Chem. 186, 90-96 (2015).

18. Bauernfeind, J. C. Carotenoid vitamin A precursors and analogs in foods and feeds. J. Agric. Food Chem. 20, 456-473 (1972).

19. Gruber, K. Agrobiodiversity: the living library. Nature 544, S8-S10 (2017).

20. Drapal, M. et al. Identification of metabolites associated with water stress responses in Solanum tuberosum L. clones. Phytochemistry 135, 24-33 (2017).

21. Price, E. J., Wilkin, P., Sarasan, V. \& Fraser, P. D. Metabolite profiling of Dioscorea (yam) species reveals underutilised biodiversity and renewable sources for high-value compounds. Sci. Rep. 6, 29136 (2016).

22. Rodriguez-Bonilla, L. et al. Assessment of genetic diversity of sweet potato in Puerto Rico. PLoS One 9, e116184 (2015).

23. Roullier, C., Rossel, G., Tay, D., McKey, D. \& Lebot, V. Combining chloroplast and nuclear microsatellites to investigate origin and dispersal of New World sweet potato landraces. Mol. Ecol. 20, 3963-3977 (2011).

24. Roullier, C., Benoit, L., McKey, D. B. \& Lebot, V. Historical collections reveal patterns of diffusion of sweet potato in Oceania obscured by modern plant movements and recombination. Proc. Natl. Acad. Sci. U.S.A. 110, 2205-2210 (2013).

25. Kyndt, $\mathrm{T}$. et al. The genome of cultivated sweet potato contains Agrobacterium T-DNAs with expressed genes: an example of a naturally transgenic food crop. Proc. Natl. Acad. Sci. U.S.A. 112, 5844-5849 (2015).
26. Park, S.-C. et al. Enhanced accumulation of carotenoids in sweetpotato plants overexpressing IbOr-Ins gene in purple-fleshed sweetpotato cultivar. Plant Physiol. Biochem. 86, 82-90 (2015).

27. Li, L., Paolillo, D. J., Parthasarathy, M. V., DiMuzio, E. M. \& Garvin, D. F. A novel gene mutation that confers abnormal patterns of $\beta$-carotene accumulation in cauliflower (Brassica oleracea var. botrytis). Plant J. 26, 59-67 (2001).

28. Grüneberg, W. J., Mwanga, R., Andrade, M. \& Espinoza, J. in Plant Breeding and Farmer Participation (eds S. Ceccarelli, S. et al.) Ch. 13, 275-322 (FAO, Rome, Italy 2009).

29. Tumwegamire, S. et al. Evaluation of dry matter, protein, starch, sucrose, $\beta$-carotene, iron, zinc, calcium, and magnesium in East African sweetpotato [Ipomoea batatas (L.) Lam] germplasm. HortScience 46, 348-357 (2011).

30. Cervantes-Flores, J. C. et al. Development of a genetic linkage map and identification of homologous linkage groups in sweetpotato using multipledose AFLP markers. Mol. Breed. 21, 511-532 (2008).

31. Medicine, I. O. Dietary Reference Intakes for Vitamin A, Vitamin K, Arsenic, Boron, Chromium, Copper, lodine, Iron, Manganese, Molybdenum, Nickel, Silicon, Vanadium, and Zinc (The National Academies Press, Washington (DC), United States 2001).

32. Grace, M. H. et al. Phytochemical changes in phenolics, anthocyanins, ascorbic acid, and carotenoids associated with sweetpotato storage and impacts on bioactive properties. Food Chem. 145, 717-724 (2014).

33. zum Felde, T. et al. In Proc. 15th International Symposium of the International Society for Tropical Root Crops (ISTRC) 27-33 (Lima, Peru, 2009).

34. Nogueira, M., Mora, L., Enfissi, E. M., Bramley, P. M. \& Fraser, P. D. Subchromoplast sequestration of carotenoids affects regulatory mechanisms in tomato lines expressing different carotenoid gene combinations. Plant Cell 25, 4560-4579 (2013).

35. Fraser, P. D., Pinto, M. E., Holloway, D. E. \& Bramley, P. M. Technical advance: application of high-performance liquid chromatography with photodiode array detection to the metabolic profiling of plant isoprenoids. Plant J. 24, 551-558 (2000).

36. Enfissi, E. M. A. et al. Integrative transcript and metabolite analysis of nutritionally enhanced DE-ETIOLATED1 downregulated tomato fruit. Plant Cell 22, 1190-1215 (2010).

37. Shahaf, N. et al. Constructing a mass measurement error surface to improve automatic annotations in liquid chromatography/mass spectrometry based metabolomics. Rapid Commun. Mass Spectrom. 27, 2425-2431 (2013).

38. Wehrens, R., Bloemberg, T. G. \& Eilers, P. H. Fast parametric time warping of peak lists. Bioinformatics 31, 3063-3065 (2015).

39. Xia, J. and Wishart, D.S. 2016. Using MetaboAnalyst 3.0 for comprehensive metabolomics data analysis. Curr. Protoc. Bioinform. 55:14.10.1-14.10.91. https://doi.org/10.1002/cpbi.11 